\title{
Case Report \\ Femoral hypoplasia - unusual facies syndrome, presentation of rare case
}

Volume 6 Issue I - 2017

\section{Introduction}

Femoral Hypoplasia-Unusual Facies syndrome (FH-UFS) is a rare, sporadic syndrome of unknown etiology, with a prevalence of $<1 \backslash 1000000$. A review of the literature reveals that there are few reported cases of syndromes similar to our case, with a preponderance of female gender to be affected. ${ }^{1,2}$ To date, 56 cases have been reported in the literature, last of which was in Bangladish in 2014. ${ }^{3,4}$ This was recognized as a distinct entity in 1975 by Daentl et al. who reported their cases of femoral hypoplasia - unusual facies syndrome, but this cases was first prescribed by Franz and O"Rahilly in 1961.,56 There has been speculation of a link to maternal Diabetes Mellitus. ${ }^{7,8}$

This syndrome encompasses a spectrum of anomalies including bilateral femoral hypoplasia and cranio-facial dysmorphism with cleft palate and other several systemic anomalies like short or absent fibulas, shortening of humeri, restricted motion of elbows, constricted ilial base, vertical ischial axis, hypoplastic acetabulae, large obturator foramina, lower spine abnormalities and posterior tapering of the ribs. In addition, there is shortening of the femur leading to thigh and leg length discrepancy and talipes equinovarus deformity. ${ }^{1,7}$

Keywords: hypoplasia of the femurs, cranio-facial dysmorphism

\section{Case history}

Our case is the second child of healthy non-consanguineous parents, delivered by Lower Segment Caesarean Section (LSCS) route at 38th week of gestation to a 21 years old mother booked in our hospital. The mother is a case of type I diabetes mellitus on insulin.

The antenatal ultrasound examination (Figure 1) revealed a fetus with bi-parietal diameter and abdominal parameter consistent with 20 weeks gestation, bilateral abnormal widening of the lower spine was demonstrated, and the sacrum was not visualized. The most significant abnormalities involved the lower extremities. both femoral bones are noted visualised, however on the right side a suspicion of a small hyperplastic bone is seen proximal to the tibia measures about 1 $\mathrm{cm}$ in length, otherwise the rest of long bones at both lower and upper limbs are within normal. The amniotic fluid volume was subjectively increased. The intracranial anatomy appeared normal, the kidneys and bladder were normal. Amniocentesis was done in view of abnormal antenatal ultrasound and reported as normal male fetus. The first sibling was a normal full term baby.

The birth weight was $2.440 \mathrm{Kg}$ ( $<3$ rd centile), Head Circumference: $34.6 \mathrm{~cm}$ (10th - 50th centile), the baby was pink with good perfusion maintaining his oxygen saturation on room air, active, with minimal tachypnea.

The following clinical findings were noted: craniofacial dysmorphism, low set ears, low hair line, upslanted palpebral fissures,

\author{
Elsayed Amany A,' El-Atawi Khaled M, ${ }^{2}$ \\ Elhalik Mahmoud S, ${ }^{3}$ Bastaki Fatma $\mathrm{A}^{4}$ \\ 'Specialist Neonatologist, Latifa Hospital, U.A.E \\ ${ }^{2}$ Consultant Neonatologist, Latifa Hospital, U.A.E \\ ${ }^{3}$ Consultant Neonatologist and Head of Paediatric Department \\ Latifa Hospital, U.A.E \\ ${ }^{4}$ Consultant Pediatrician/ Clinical Genetics and Metabolic, Latifa \\ Hospital, U.A.E
}

Correspondence: Amany A. Elsayed, Specialist Neonatologist, Latifa Hospital, Po Box 29793 Dubai, United Arab Emirates, Tel 972000000000,Email amany.abdelsamed.@yahoo.com

Received: August 07, 2016 | Published: January 02, 2017

long philtrum with thin upper lip, micrognathia, hypoplastic alae nasi and cleft palate, bilateral hypoplasia of femur with dyplastic hips, showing flexion and adduction deformity. Length of the thigh was very short compared to the length of the leg, with a knee joint being rudimentary. Bilateral talipes equinovarus deformity of varying degrees (Figures 2\&3).

The following Investigations were done: Karyotyping was normal male (46 XY), 2D Echocardiography showed tiny Patent Ductus Arteriousus (PDA), Brain Ultrasound and Abdominal Ultrasound revealed normal findings. Ultrasound of both hips showed undeveloped acetabulum, the chondral tibial epiphyses of both tibia appears larger in size (approximately $17 \mathrm{~mm}$ x 17mm), resembling the size of normal femoral chondral epiphyses, and the tibia seems forming pseudo sockets in the soft tissue outside the iliac bones. His Skeletal Survey (Figure 4) the Skull showed that the facial bones are smaller in size compared with cranial bones, and a small sized anterior fontanel, the Spine were with normal appearance, the chest showed a thin ribs and clavicles, the pelvis showed that the distance between the ischial bones is widened, the long bones revealed bilateral absent femurs and thin osteoporotic fibulae while the small bones were overlapped.

In view of dysmorphic features, Exome Sequencing test was done but no clinically significant variants were identified through analysis of whole sequencing data, which confirm the finding suggestive of femoral hypoplasia unusual faces syndrome. The baby was seen by maxillofacial team in view of bilateral choanal stenosis for which bilateral nasal tubes were inserted (Figure 2). Baby was discharged at the age of 15 days, with follow up appointments to multidisciplinary clinics; Genetic counselling may be of benefit for patients and their families. Other treatment is symptomatic and supportive.

\section{Discussion}

Femoral Hypoplasia - Unusual Facies Syndrome includes bilateral femoral hypoplasia, facial dymorphisim with cleft palate, micrognathia, long philtrum, thin upper lip with short broad tipped nose and dysplasia of hips. Other malformations including skeletal 
defects and visceral abnormalities in cardiovascular and genitourinary systems may be found. ${ }^{9,10}$

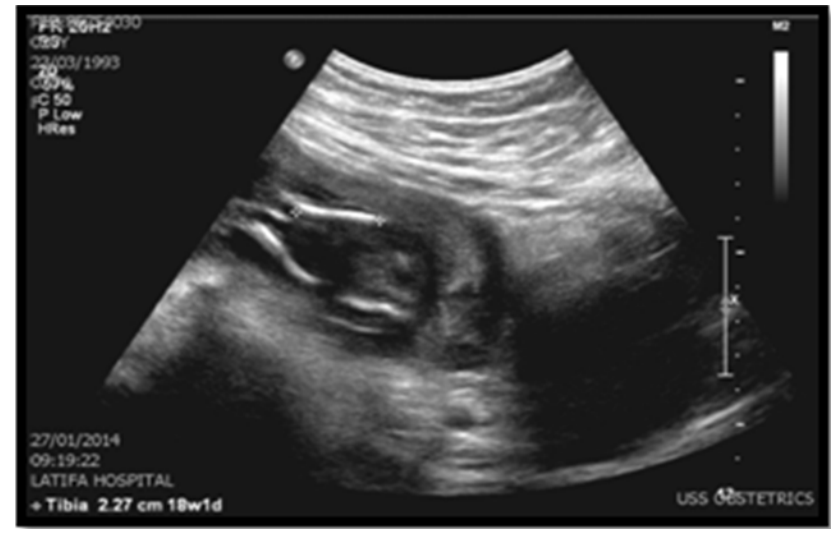

Figure I Antenatal ultrasound showing both femoral bones are noted visualised.

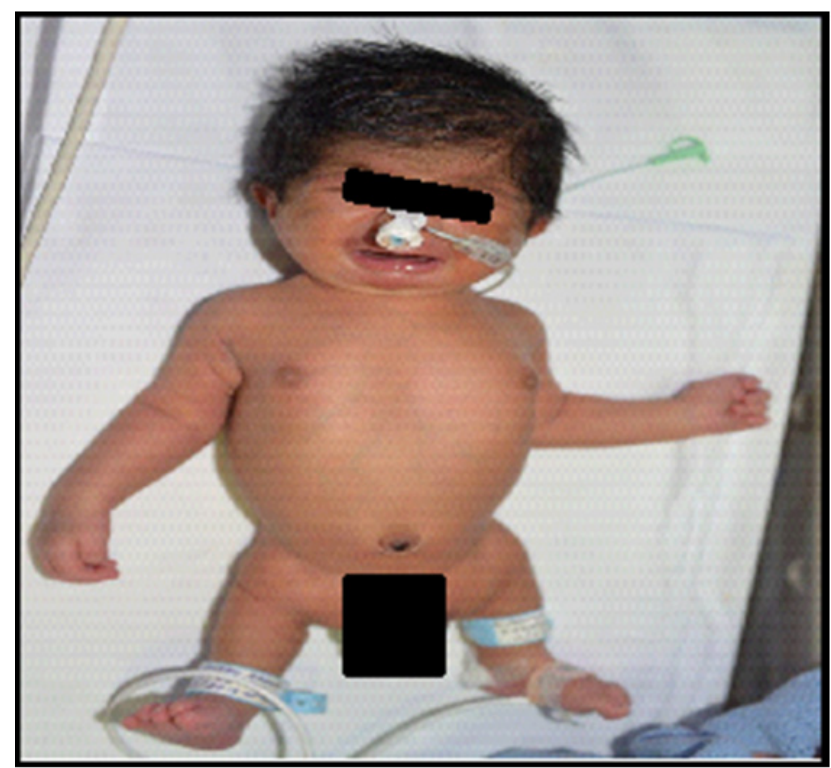

Figure 2 Bilateral absent femur with abnormal facies.

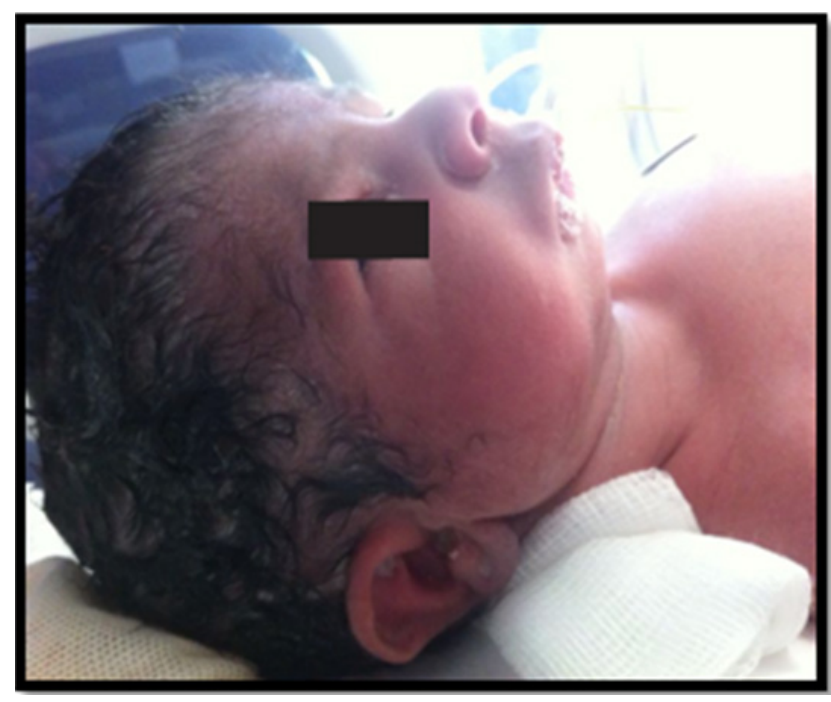

Figure 3 Low set ears with micrognathia.

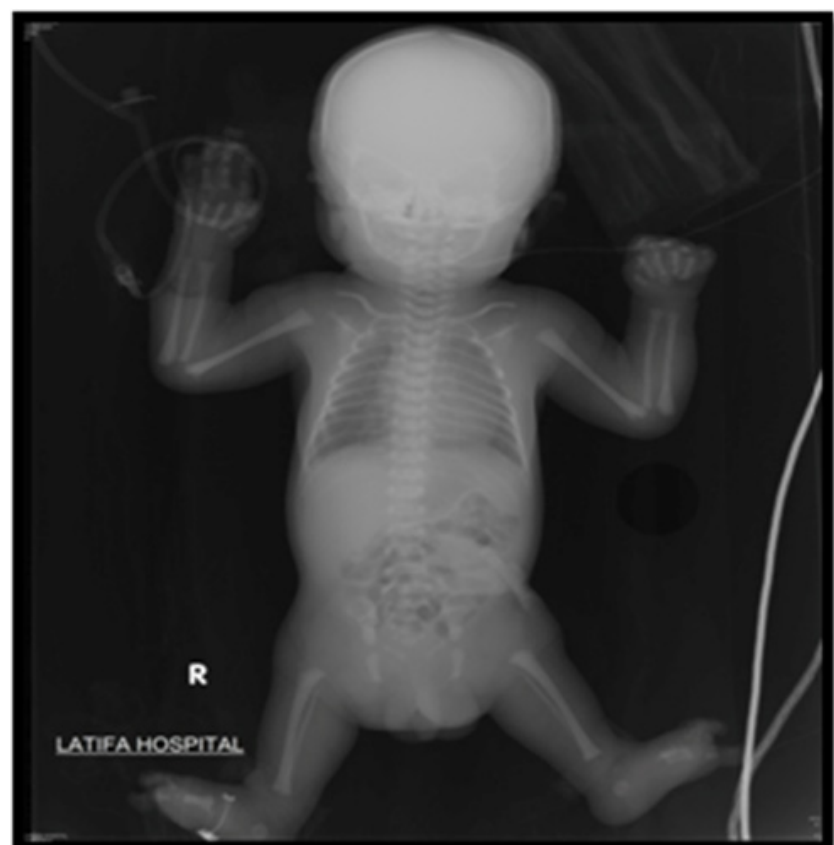

Figure 4 Skeletal survey showing absent femur.

As the maternal insulin does not cross the placenta, and fetal insulin is not detectable until 10 weeks, raising the possibility of maternal diabetes or glucose homeostasis being the teratogenic factor in femoral hypoplasia - unusual facies syndrome. ${ }^{6}$ However, craniofacial anomalies have been rarely reported in such infants. ${ }^{11}$

Similarly, in our case, there was maternal diabetes and our clinical findings showed similar malformations with craniofacial dysmorphisim, bilateral femoral hypoplasia, micrognathia, upper thin lip, cleft palate and congenital heart disease like patent ductus arteriosus. With this framework, the case fits into classical clinical spectrum of FH-UFS.

Regarding the outcome there may be problems in feeding and speech development but many patients have been of normal to above normal intelligence and most of them have been ambulatory., ${ }^{72}$ Most complications arise from small stature and limited function of the lower limbs. ${ }^{7}$ However, most patients are ambulatory. ${ }^{5}$ Recurrent urinary tract infection and incontinence have also been reported. ${ }^{8}$ However, in cases without serious complications, the life span is usually normal. ${ }^{8}$

When detected before viability, the option of termination of pregnancy can be offered to the parent. The postnatal management is directed at the orthopedic, facial, cardiac and genitourinary complications.

\section{Acknowledgements}

None.

\section{Conflict of interest}

The authors declare no conflict of interest.

\section{Funding}

None. 


\section{References}

1. Burck U, Riebel T, Held KR, et al. . Bilateral femoral dysgenesis with micrognathia, cleft palate, anomalies of the spine and pelvis, and foot deformities. Helv Paediat Acta. 1981;36(5):473-482.

2. Pitt DB, Findlay II, Cole WG, et al. Case report:Femoral hypoplasiaunusual facies syndrome. Aust Paediatr J. 1982;18(1):63-66.

3. http://www.orpha.net/consor/cgi-bin/Disease_Search. php? lng=EN\&data id=1928\&Disease Disease Search diseaseGroup=femoral-hypoplasia\&Disease_Disease_Search disease Type $=$ Pat $\&$ disease $(\mathrm{s}) /$ group $\% 200 \mathrm{o} \% 20 \overline{\text { diseases }}=\overline{\text { Femoral }}-$ hypoplasia--- unusual-facies - syndrome $\&$ title $=$ Femoral hypoplasia__ unusual-facies-syndrome\&search=Disease_Search Simple

4. Begum Sharifun Naher. Fraser Syndrome - A Case Report. Bangladesh $J$ Child Health. 2014;38(2):50.

5. Say B. Femoral hypoplasia-unusual facies syndrome. In: Buyse ML (Ed):Birth defect encyclopedia, Blackwell Scientific Publication, Dover, MA. 1990. p. 681 .
6. Lampert RP. Dominant inheritance of femoral hypoplasia - unusual facies syndrome. Clin Genet. 1980;17(4):255-258.

7. Daentl DL, Smith DW, Scott CI, et al. Femoral hypoplasia-unusual facies syndrome. J Pediatr. 1975;86:107-111.

8. Jones KL. Smith"s Recognizable patterns of human malformation. 4th ed. WB Saunders, Philadelphia. 1988. p. 268-269.

9. Burn J, Winter RM, Baraitser M, et al. The femoral hypoplasia - unusual facies syndrome. J Med Genet. 1984;21:331-340.

10. Nowaczyk MJ, Huggins MJ, Fleming A, et al. Femoral-facial syndrome:prenatal diagnosis and clinical features. Report of three cases. Am J Med Genet A. 2010;152A(8):2029-2033.

11. Ewart-Toland A, Yankowitz J, Winder A, et al. Oculoauriculovertebral abnormalities in children of diabetic mothers.American journal of Medical Genetics. 2000;90(4):303-309.

12. DePalma L, Huray PH, Popeo VR. Femoral hypoplasisa - unusual facies syndrome:Autopsy findings in an unusual case. Pediatr Pathol. $1986 ; 5(1): 1-8$. 